\title{
PRESSURE-DRIVEN DEMAND AND LEAKAGE SIMULATION FOR WATER DISTRIBUTION NETWORKS
}

\author{
Orazio Giustolisi ${ }^{1}$, Dragan Savic ${ }^{2}$ and Zoran Kapelan ${ }^{3}$
}

Manuscript no: HYENG-07-6263

Corresponding Author:

Professor Orazio Giustolisi

${ }^{1}$ Professor, Technical University of Bari, Dean, II Engineering Faculty, Dep. of Civil and Environmental Eng., via Turismo, 8, 74100 Taranto, Italy, phone +39 3293173094, fax: +39 0805963719, email: o.giustolisi@poliba.it

${ }^{2}$ Professor, University of Exeter, Centre for Water Systems, Harrison Building, North Park Road, EX4 4QF, Exeter, United Kingdom, phone: +44 1392 263637, fax: +44 1392 217965, email: $\underline{\text { d.savic@ex,ac,uk }}$

${ }^{3}$ Senior Lecturer, University of Exeter, Centre for Water Systems, Harrison Building, North Park Road, EX4 4QF, Exeter, United Kingdom, phone: +44 1392 263730, fax: +44 1392 217965, email: z.kapelan@ex,ac,uk 


\title{
PRESSURE-DRIVEN DEMAND AND LEAKAGE SIMULATION FOR WATER DISTRIBUTION NETWORKS
}

\begin{abstract}
Increasingly, water loss via leakage is acknowledged as one of the main challenges facing water distribution system operation. The consideration of water loss over time, as systems age, physical networks grow and consumption patterns mature, should form an integral part of effective asset management, rendering any simulation model capable of quantifying pressure-driven leakage indispensable. To this end, a novel steady-state network simulation model that fully integrates, into a classical hydraulic representation, pressure-driven demand and leakage at the pipe level is developed and presented here. After presenting a brief literature review about leakage modelling, the importance of a more realistic simulation model allowing for leakage analysis is demonstrated. Then, the algorithm is tested from a numerical standpoint and subjected to a convergence analysis. These analyses are performed on a case study involving two networks derived from real systems. Experimentally observed convergence/error statistics demonstrate the high robustness of the proposed pressure-driven demand and leakage simulation model.
\end{abstract}

\section{KEYWORDS}

Pressure-driven demands, leakages, leakage model, pipe deterioration, pipe network. 


\section{INTRODUCTION}

Water loss via leakage constitutes a major challenge to the effective operation of municipal distribution networks since it represents not only diminished revenue for utilities, but also undermined service quality (Almadoz et al., 2005) and wasted energy resources (Colombo and Karney, 2002). A typical leakage control program usually starts with a water audit based on available flow measurements. Although this is an important first step, most practical studies do not go beyond it. In order to assist in leakage reduction and conduct more accurate analysis, a hydraulic model capable of accounting for pressure-driven (also known as head-driven) demand and leakage flow at pipe level should prove invaluable, and extended period simulation should be involved.

In fact, an integral part of any medium to long term rehabilitation planning for a water distribution system should be the analysis of pipe deterioration and the corresponding increase in water loss. In order to obtain a better estimate of flow through the network (with respect to both satisfied demand and losses through leakage), a pressure-driven hydraulic model is needed. Several models have been developed to incorporate pressure-driven demand analysis into network reliability evaluation (Chandapillai, 1991; Gupta and Bhave, 1996; Ackley et al., 2001; Kalungi and Tanyimboh, 2003; Wu et al., 2006). This paper introduces a new simulation model that fully integrates a classic hydraulic simulation algorithm, such as that of Todini and Pilati (1988) found in EPANET 2 (Rossman, 2000), with a pressure-driven model that entails a more realistic representation of leakage (Germanopoulos, 1985; Germanopoulos and Jowitt, 1989). The novel pressure-driven algorithm uses the framework of Todini's simulation model (Todini, 2003) in order to account for pressure-driven leakage at the pipe level and offer a more realistic representation. 


\section{PRESSURE-DRIVEN DEMAND/LEAKAGE NETWORK SIMULATION MODEL}

The pressure-driven simulation of a network comprising $n_{p}$ pipes with unknown discharges (i.e., flow rates), $n_{n}$ nodes with unknown heads (internal nodes or junctions in the EPANET terminology) and $n_{0}$ nodes with known heads (tank levels, for example) can be described as in Todini (2003). Therefore, assuming the elements of the diagonal matrix $\mathbf{A}_{p p}$ of order $n_{p}$ equal to $R_{k}\left|Q_{k}\right|^{n-}$ ${ }^{1}$, the pressure-driven simulation of a network can be described by the following system of equations based on energy and mass balance conservation:

$\left[\begin{array}{ll}\mathbf{A}_{p p} & \mathbf{A}_{p n} \\ \mathbf{A}_{n p} & \mathbf{A}_{n n}\end{array}\right]\left[\begin{array}{l}\mathbf{Q} \\ \mathbf{H}\end{array}\right]=\left[\begin{array}{c}-\mathbf{A}_{p 0} \mathbf{H}_{0} \\ \mathbf{0}\end{array}\right]$

where:

$\mathbf{Q}=\left[Q_{1}, Q_{2}, \ldots, Q_{n p}\right]^{T}$ is the $\left[n_{p}, 1\right]$ column vector of unknown pipe flow rates;

$\mathbf{H}=\left[H_{1}, H_{2}, \ldots, H_{n n}\right]^{T}$ is the $\left[n_{n}, 1\right]$ column vector of unknown nodal heads;

$\mathbf{H}_{0}=\left[H_{01}, H_{02}, \ldots, H_{0 n 0}\right]^{T}$ is the $\left[n_{o}, 1\right]$ column vector of known nodal heads;

In the system of Equations (1), $\mathbf{A}_{p n}=\mathbf{A}_{n p}^{T}$ and $\mathbf{A}_{p 0}$ are topological incidence sub-matrices of size $\left(n_{p}, n_{n}\right)$ and $\left(n_{p}, n_{0}\right)$, respectively, derived from the general topological matrix $\overline{\mathbf{A}}_{p n}=\left[\begin{array}{lll}\mathbf{A}_{p n} & \mathbf{A}_{p 0}\end{array}\right]$ of size $\left[n_{p}, n_{n}+n_{0}\right]$, as defined in (Todini, 2003).

In order to account for leakage flow rates in model (1), $\mathbf{A}_{n n}$ can be cast as a diagonal matrix whose elements are the scalar product (i.e. element by element product) $-\left(\mathbf{q}_{\text {act }}+\mathbf{q}_{l}\right) \mathbf{H}^{-1}$. $\mathbf{q}_{a c t}(\mathbf{H})=\left[q_{1-a c t}\left(H_{1}\right), q_{2-a c t}\left(H_{2}\right), \ldots, q_{n n-a c t}\left(H_{n n}\right)\right]^{\mathrm{T}}$ is the column vector of pressure/head-driven nodal demands and $\mathbf{q}_{l}$ is the column vector of nodal leakage flow rates (assuming positive sign for node discharge). Actually, leakage occurs at pipe level and is just reported at the nodes as will be explained subsequently. The column vector $\mathbf{0}$ in the system (1) is related to the fact that the ma- 
trix $\mathbf{A}_{n n}$ (by means of the vectors $\mathbf{q}_{a c t}$ and $\mathbf{q}_{l}$ ) contains the pressure-driven demands and leakage flows.

For reasons of simplicity, while still preserving generality, elements such as pumps, valves and other dissipation devices have not been considered in the simulation model (1). Furthermore, the single expression is used to define the head loss along the $k$-th pipe in the network, regardless of the flow regime assumed and the head loss relationship used. Therefore, $R_{k}$ is the head loss coefficient that is a function of a pipe's roughness, diameter and length, while $n$ is an exponent which takes into account the actual flow regime and the head loss relationship used (1.85 for the HazenWilliams and 2 for the Darcy-Weisbach models).

In the system of Equations (1), $\mathbf{q}_{a c t}$ is the column vector characterizing demand-pressure dependence whose elements are defined for the $i$-th node of the network by the function $q_{i-a c t}\left(H_{i}\right)$ or $q_{i-}$ ${ }_{a c t}\left(P_{i}\right)$ (see below). For $q_{i-a c t}\left(P_{i}\right)$ the following relationship will be used here (Wagner et al., 1988):

$q_{i-a c t}=\left\{\begin{array}{cc}q_{i-\text { design }} & \text { for } P_{i}>P_{i-\text { ser }} \\ q_{i-\text { design }}\left(\frac{P_{i}-P_{i-\min }}{P_{i-\text { ser }}-P_{i-\min }}\right)^{1 / 2} & \text { for } P_{i-\min } \leq P_{i} \leq P_{i-\text { ser }} \\ 0 & \text { for } P_{i}<P_{i-\min }\end{array}\right.$

where $P_{i \text {-ser }}$ is the minimum service pressure required for supplying demand $q_{i \text {-design }}$ (those used for network design purposes, for example) and $P_{i-\min }$ denotes the range of the intermediate work- 
ing condition when the actual demand is given as $q_{i-a c t}$. For demands that are not pressure-driven, Equations (2) become $q_{i-a c t}=q_{i-d e s i g n}$.

At the scale of individual pipes, the pressure-leakage relationship is defined in $\mathbf{q}_{l}$, a column vector whose elements are nodal leakages $q_{i-l e a k}$ that is computed from the pipe leakage model $q_{k \text {-leak }}$. In order to avoid confusion among variables, the index $i$ will be used for nodal-level variables and $k$ for pipe-level variables. Thus, assuming a uniform distribution of leakage $q_{k \text {-leak }}$ along pipe $k$, the background leakage model can be expressed as (Germanopoulos, 1985; Germanopoulos and Jowitt, 1989):

$q_{k-\text { leak }}= \begin{cases}\beta_{k} l_{k}\left(P_{k}\right)^{\alpha_{k}} & \text { if } P_{k}>0 \\ 0 & \text { if } P_{k} \leq 0\end{cases}$

where $P_{k}$ is the average pressure in the pipe computed as the mean of the pressure values at the end nodes $i$ and $j$ of the $k$-th pipe, and $l_{k}$ is the length of that pipe. Variables $\alpha_{k}$ and $\beta_{k}$ denote the two leakage model parameters (explained and discussed later in the text). The average pressure vector $\mathbf{P}^{\text {pipes }}$ can be computed from the general topological matrix and nodal pressure as:

$\mathbf{P}^{\text {pipes }}=\frac{\left(\left|\overline{\mathbf{A}}_{p n}\right|\left[\mathbf{P}^{\text {nodes }}{ }_{\mid} \mathbf{P}_{0}^{\text {nodes }}\right]\right)}{2}$

where $\mathbf{P}^{\text {nodes }}$ is the pressure vector of unknown nodal heads and $\mathbf{P}_{0}{ }^{\text {nodes }}$ is the pressure vector of known nodal heads $\left(\left|\overline{\mathbf{A}}_{p n}\right|\right.$ is the absolute value of the topological matrix).

The allocation of leakage to the two end nodes can be performed in a number of ways. The simplest approach assumes that half of total leakage from the pipe element occurs at each of the end nodes. A more realistic approach, however, divides the total leakage in proportion with the magnitude of the two nodal pressures (Ainola et al., 2000). With either of these two approaches, the 
nodal leakage flow $q_{i \text {-leak }}$ is computed as the sum of $q_{k \text {-leak }}$ flows of all pipes connected to node $i$ as follows:

$q_{i-\text { leak }}=\sum_{k} \frac{1}{2} q_{k-\text { leak }}=\frac{1}{2} \sum_{k} \beta_{k} l_{k}\left(P_{k}\right)^{\alpha_{k}}$

or

$q_{i-\text { leak }}=\sum_{k} \frac{P_{i}}{P_{i}+P_{j}} q_{k-\text { leak }}=\frac{P_{i}}{2} \sum_{k} \beta_{k} l_{k}\left(P_{k}\right)^{\alpha_{k}-1}$

where $P_{k}=\frac{P_{i}+P_{j}}{2}$

Thus, the elements of the vector $\mathbf{q}_{l}$ can be computed from the topological matrices as follows:

$\mathbf{q}_{l}=\left(\frac{1}{2}\right.$ or $\left.\frac{P_{i}}{2}\right) a b s\left(\mathbf{A}_{n p}\right)\left[\begin{array}{c}q_{1-\text { leak }} \\ 0 \\ q_{k-\text { leak }} \\ 0 \\ q_{n p-\text { leak }}\end{array}\right]$
$q_{k-\text { leak }}=\left\{\begin{array}{cc}\beta_{k} l_{k}\left(P_{k}\right)^{\alpha_{k}} \text { or } \beta_{k} l_{k}\left(P_{k}\right)^{\alpha_{k}-1} & \text { if } P_{k}>0 \\ 0 & \text { if } P_{k} \leq 0\end{array}\right.$

where diag stands for the diagonal elements of the matrix. Thus, the $\mathbf{q}_{l}$ elements are from the matrix product involving topological matrix $\mathbf{A}_{n p}$ (i.e. $\mathbf{A}_{p n}$ ). The solution to the system of Equations (1) for the pressure-driven demands defined in (2) was first given by Todini (2003). Here, the procedure is further expanded to account for pressure-driven leakage and consider large networks. The procedure involves the initialisation phase followed by the iteration phase as specified in the following equations:

$\underline{\text { Initialisation phase }}$ 


$$
\begin{aligned}
& \mathbf{\Lambda}_{p p}^{i t e r=0}=\mathbf{I}_{p p} \\
& \Lambda_{n n}^{i t e r=0}=\mathbf{I}_{n n} \\
& \mathbf{H}^{i t e r=0}=\mathbf{P}_{s e r}+\mathbf{H} \mathbf{L} \\
& \mathbf{Q}^{i t e r=0}=\left(\mathbf{R}_{p p}\right)^{-1}
\end{aligned}
$$

where $\mathbf{I}_{n n}$ and $\mathbf{I}_{p p}$ are identity matrices; $\boldsymbol{\Lambda}_{n n}$ and $\boldsymbol{\Lambda}_{p p}$ are diagonal matrices of over-relaxation coefficients for nodal heads and pipe flow updating; $\mathbf{R}_{p p}$ is a diagonal matrix whose elements are $R_{k}$; HL is the vector of nodal elevations; $\mathbf{P}_{s e r}$ is a vector whose elements are $P_{i-s e r}$. The initialisation part of the algorithm shown in Equation (7a) ensures that flows $\mathbf{Q}^{\text {iter }=0}$ make $\mathbf{D}_{p p}=\mathbf{I}_{p p}$ for iter $=0$.

\section{Iteration phase}

$$
\begin{aligned}
& \mathbf{A}^{i t e r}=\mathbf{A}_{n p}\left(\mathbf{D}_{p p}^{i t e r}\right)^{-1} \mathbf{A}_{p n}-\left(\mathbf{D}_{n n}^{i t e r}+\mathbf{D} \mathbf{L}_{n n}^{i t e r}\right) \\
& \mathbf{F}^{i t e r}=\left[\mathbf{A}_{n p} \mathbf{Q}^{i t e r}-\left(\mathbf{q}_{a c t}^{i t e r}+\mathbf{q}_{l}^{i t e r}\right)\right]-\mathbf{A}_{n p}\left(\mathbf{D}_{p p}^{i t e r}\right)^{-1}\left(\mathbf{A}_{p 0} \mathbf{H}_{0}+\mathbf{A}_{p p}^{i t e r} \mathbf{Q}^{i t e r}\right)-\left(\mathbf{D}_{n n}^{i t e r}+\mathbf{D} \mathbf{L}_{n n}^{i t e r}\right) \mathbf{H}^{i t e r} \\
& \mathbf{H}^{i t e r+1}=\left(\mathbf{A}^{i t e r}\right)^{-1} \mathbf{F}^{i t e r} \\
& \mathbf{Q}^{i t e r+1}=\mathbf{Q}^{i t e r}-\left(\mathbf{D}_{p p}^{i t e r}\right)^{-1}\left(\mathbf{A}_{p p}^{i t e r} \mathbf{Q}^{i t e r}+\mathbf{A}_{p n} \mathbf{H}^{i t e r+1}+\mathbf{A}_{p 0} \mathbf{H}_{0}\right) \\
& \mathbf{H}^{i t e r+1}=\boldsymbol{\Lambda}_{n n}^{i t e r}\left(\mathbf{H}^{i t e r+1}-\mathbf{H}^{i t e r}\right)+\mathbf{H}^{i t e r} \\
& \mathbf{Q}^{i t e r+1}=\mathbf{\Lambda}_{p p}^{i t e r}\left(\mathbf{Q}^{i t e r+1}-\mathbf{Q}^{i t e r}\right)+\mathbf{Q}^{i t e r}
\end{aligned}
$$

In the iterative part of the algorithm, $\mathbf{D}_{n n}, \mathbf{D L}_{n n}$ and $\mathbf{D}_{p p}$ are diagonal matrices whose elements denote derivatives of $\mathbf{q}_{a c t}, \mathbf{q}_{l}$ elements and $R_{k}\left|Q_{k}\right|^{\mathrm{n}-1} Q_{k}$ with respect to nodal pressure, pipe pressure and pipe flow, respectively. For example, $\mathbf{D}_{n n}$ is the $\left(n_{n}, n_{n}\right)$ diagonal matrix given as: 
$\mathbf{D}_{n n}(i, i)=\left\{\begin{array}{cc}0 & \text { for } P_{i}>P_{i-\text { ser }} \\ \frac{q_{i-\text { design }}}{2} \frac{\left(P_{i}-P_{i-\min }\right)^{-1 / 2}}{\left(P_{i-\text { ser }}-P_{i-\text { min }}\right)^{1 / 2}} & \text { for } P_{i-\text { min }} \leq P_{i} \leq P_{i-\text { ser }} \\ 0 & \text { for } P_{i}<P_{i-\text { min }}\end{array}\right.$

and $\mathbf{D} \mathbf{L}_{n n}$ represents a $\left(n_{n}, n_{n}\right)$ diagonal matrix whose elements can be calculated from the derivatives of $q_{k \text {-leak }}$ with respect to $P_{k}$ (see Equation (5)). Similarly to matrix $\mathbf{q}_{l}$ in Equation (6), elements of matrix $\mathbf{D L}_{n n}(i, i)$ can be obtained as follow:

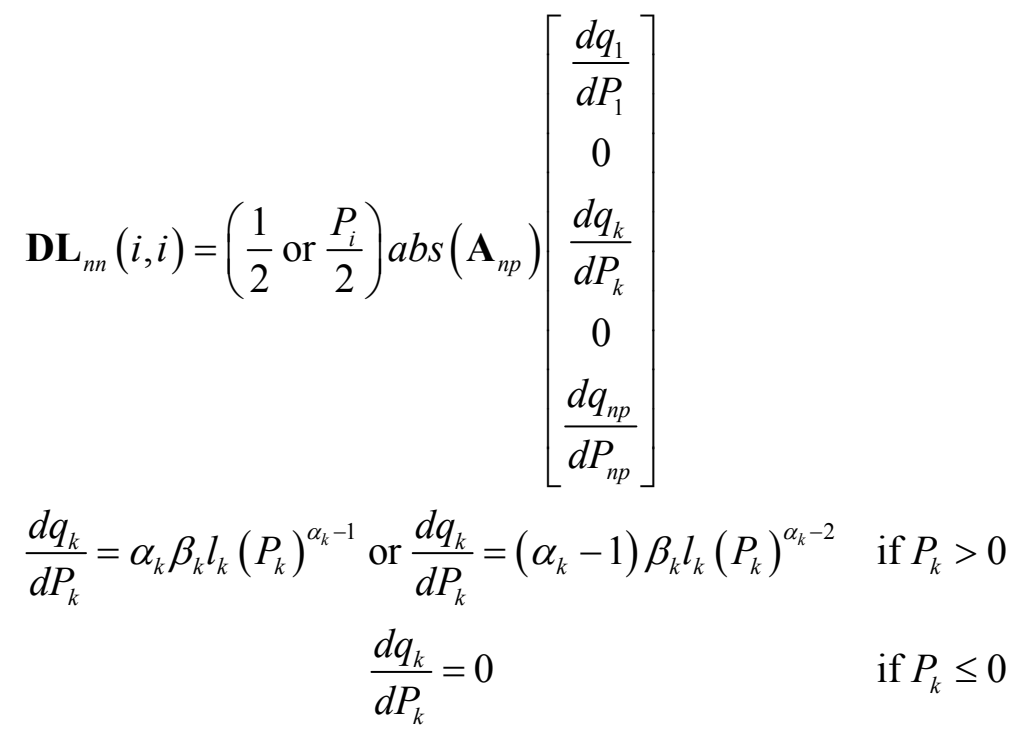

Note that both $\mathbf{q}_{l}$ and $\mathbf{D} \mathbf{L}_{n n}(i, i)$ elements can be constructed using the scalar formulation, as done by Rossman (2000) for the elements of matrix $\mathbf{A}(i, i)$ (see page 188 in the EPANET 2 Users Manual) or Todini (2003). 
Moreover, the diagonal elements of both $\Lambda$ matrices are assumed equal to $\lambda^{i \text { iter }}$ (a real number in the $[0,1]$ range) which works as a step size, or over-relaxation coefficient, for updating nodal heads and pipe flow rates across iterations. It is worth noting that $\lambda^{\text {iter }=0}$ is set to 1 , meaning that the relaxation coefficient is not used initially, but its adaptation to the error surface (during the iterative search for the solution) is invoked if required to improve convergence behaviour. Here, the value of $\lambda^{\text {iter }}$ is driven by the mean of squared errors or by the maximum errors in the mass and energy balance equations while performing the iterative search. When any of these errors decreases, the value of $\lambda^{\text {iter }}$ increases by a factor of 5 and when any of these errors increases, the value of $\lambda^{\text {iter }}$ is reduced by a factor of 10 . If during the iterative search $\lambda^{\text {iter }}$ becomes equal to or less than the error tolerance level (e.g., $10^{-7}$ ) or mean of squared errors (e.g., $10^{-7}$ ) the search process is stopped because convergence has been reached. Experiments carried out by the authors have shown that model runs, described below, performed without the relaxation coefficient procedure can have serious convergence problems.

Furthermore, the initialization of flows used here makes the matrix $\mathbf{D}_{p p}$ (initialized to $\mathbf{I}_{p p}$ ) well conditioned, which is particularly useful for large networks. The maximum number of iterations is also used as a further threshold control.

Finally, assuming separate expressions for background leakage, $q_{k \text {-background }}$, and burst losses, $q_{k \text { - }}$ burst, the total leakage $q_{k}$ along the pipe $k$-th can be expressed using:

1. the classical formulae of orifice flow for bursts (exponent equal to 0.5); and

2. the Germanoupulos (1985) expression for background losses.

with different meaning assigned to its constants: 


$$
\begin{cases}q_{k \text {-leak }}=\beta_{k} l_{k}\left(P_{k}\right)^{\alpha_{k}}+C_{k}\left(P_{k}\right)^{0.5} & \text { if } P_{k}>0 \\ q_{k \text {-leak }}=0 & \text { if } P_{k} \leq 0\end{cases}
$$

where $\alpha_{k}$ and $\beta_{k}$ are the two parameters of the leakage flow model related to the background losses only and $C_{k}$ is a coefficient dependent on the sum of outflow coefficients related to bursts along the pipe. Therefore, Equations (5) can be rewritten as follows:

$$
\begin{aligned}
& q_{i-\text { leak }}=\sum_{k} \frac{1}{2} q_{k-\text { leak }}=\frac{1}{2} \sum_{k} \beta_{k} l_{k}\left(P_{k}\right)^{\alpha_{k}}+C_{k}\left(P_{k}\right)^{0.5} \\
& \text { or } \\
& q_{i-\text { leak }}=\sum_{k} \frac{P_{i}}{P_{i}+P_{j}} q_{k-\text { leak }}=\frac{P_{i}}{2} \sum_{k} \beta_{k} l_{k}\left(P_{k}\right)^{\alpha_{k}-1}+C_{k}\left(P_{k}\right)^{-0.5}
\end{aligned}
$$

Note that Equations (7) and (9) can be easily modified by following the above reasoning.

\section{Leakage Model Parameters ( $\alpha$ and $\beta$ )}

Water distribution system losses may be classified in being due to: background losses (from joints, fittings and small cracks); reported bursts; and unreported bursts (Lambert, 1994).

The main explanation for growing water loss (either due to bursts or small cracks) is the general deterioration of water mains, joints and service connections. Pipe degradation, pipe parameter $\beta$, has commonly been studied as a steady monotonic process that is modified by time-varying "noise" (Kleiner and Rajani, 2002). Pipe age, diameter and material have been identified as primary variables influencing the monotonic increase in the burst rate over a number of years. The majority of statistical methods consider pipe age as the most crucial variable describing the increase in pipe failure rates over time (Shamir and Howard, 1979; Kleiner and Rajani, 2001). Furthermore, Walski and Pelliccia (1982) found diameter to be a key factor, with the failure rate of smaller diameter pipes being higher than those experienced by larger ones. Studies of common metallic pipe (e.g., cast iron, ductile iron, etc.) behaviour have been conducted to establish the 
influence of pipe material on failure rates (Kleiner and Rajani, 2002; Kettler and Goulter, 1985). Moreover, age, material and diameter are usually the only, if any, information available to many municipalities and water companies. Recently, Berardi et al. (2005) demonstrated the dependence of pipe bursts on pipe length, age and diameter using real data from UK water companies.

The value of the leakage parameter $\alpha$ in Equation (5) can be described using the FAVAD (fixed and variable area discharge) approach proposed by May (1994). In fact, May originally suggested a two component model, burst losses through a constant area hole $(\alpha=0.5)$ and background losses through an area that changes linearly with pressure $(\alpha=1+0.5=1.5)$. Thus, $\alpha$ of the leakage model (5) depends on the balance between the burst $(\alpha=0.5)$ and background $(\alpha>0.5)$ leakage flows and can be determined by means of model calibration and/or component analysis. For example, Jowitt and Xu (1990) and Vairavamoorthy and Lumbers (1998) obtained the value of $\alpha=1.18$ from field data. Later, Lambert (2001) inferred a range of $\alpha$ values ranging from 0.50 to as high as 2.50 , depending on the mixture of leaks and the dominant type of leaks (simple holes: $\alpha=0.5$; longitudinal split which opens in one dimension: as in May (1994), i.e. $\alpha=1.5$; linear-radial opening: $\alpha=2.0-2.5$ ). Plastic pipes exhibit higher $\alpha$ values because of their propensity to have longitudinal splits. Interestingly, Lambert (2001) reported $\alpha \square$ values around 1.5 regardless of the pipe material. However, it is currently held that rigid pipes such as those comprised of metal are generally characterized by lower values of $\alpha$.

Based on the above discussion, the following functional relationships can be postulated for $\alpha$ and $\beta$ : 


$$
\begin{array}{ll}
\alpha_{k}=\alpha_{k}\left(\frac{d_{k}}{s_{k}}, m_{k}\right) & 1 \leq k \leq n_{p} \\
\beta_{k}=\beta_{k}\left(a g e_{k}, d_{k}, s_{k}, m_{k}, p r_{k}, \ldots\right) & 1 \leq k \leq n_{p}
\end{array}
$$

where $m$ denotes the pipe material, $p r$ is the number of properties supplied (or connections on the main) and $s$ is the pipe wall thickness. From Equation (12) that $\beta$ depends, in general, on both pipe characteristics and various external factors (e.g., environmental conditions, traffic loading, external stress and corrosion, etc.). In contrast, $\alpha$ is a function of pipe characteristics only (material $m$ and rigidity $d / s$ ).

Clearly, the change in $\beta$ over the years is related to average pressure (Lambert, 2001). From the standpoint of functional dependency, this is accounted for by the pipe age variable while, from a physical perspective, pressure generates a fatigue effect (i.e., enhancing deterioration) similar to that of traffic loading. Thus, for a specific system, escalation in the leakage rate can be described by pipe age.

Similar to pipe breaks (Shamir and Howard 1979), an exponential formulation can be used for leakage proliferation (Walski, 1987). Thus, $\beta($ age) exponentially increases at a rate whose coefficient in the function argument is dependent on the pressure regime since the rate of system deterioration is influenced by pressure management (Lambert, 2001). The value of this coefficient for the leakage increase rate, as reported in Walski (1987), can be assumed close to zero in systems where utilities routinely conduct leak repair activities, while the coefficient of pipe break growth is a reasonable estimate for a utility without an ongoing leak detection program. 
Finally, $\beta$ is more closely related to the number of leaks (or leakage area) per unit of pipe length while $\alpha$ is more strongly related to the type of leakage (therefore to the hydraulics of leakage) as governed by pipe material (Lambert, 2001).

\section{CASE STUDIES}

The objective of the case studies is neither to perform a water balance nor to calibrate leakage model parameters, but to demonstrate the effectiveness of the proposed simulation model (coupling pressure-driven nodal demands and pipe leakage) in the context of both small and large networks. The two case studies, whose characteristics are derived from real systems (named "Network A" and "Network B", respectively), serve to:

- show that the new modelling approach provides more realistic results when applied to a classical optimal network design problem (base case for comparison). For this purpose, the hypothesis of leakage flow rates proportional to nodal demands will be discussed.

- test the new simulation model from a numerical standpoint, by analysing convergence through some performance indicators.

To demonstrate that the new modelling approach provides more realistic results than the demand-driven analysis, the two networks were optimized to obtain the least-cost design for each of them (see for example, Savic and Walters (1997) for the formulation of the optimization problem) and the solutions were tested using the pressure-driven approach. The layout of the smaller network ("Network A"), whose characteristics were derived from a real Italian system, is depicted in Figure 1 with corresponding data provided in Tables 1-3. Pipe diameters in Table 2 have been determined by minimising the total design cost subject to a minimum pressure $\mathbf{P}_{\text {ser }}$ 
(equal to $10 \mathrm{~m}$ for all the nodes) constraint for supplying the prescribed demand $\mathbf{q}_{\text {design }}$ (base case). Based on common practice where leakage is assumed to represent a percentage of nodal demand (e.g., $25 \%$ in Italy), the actual required demand was adopted for the design study as $\mathbf{q}_{d e-}$ ${ }_{\text {mand }}=\mathbf{q}_{\text {design }} / 1.25$,. The following relationship was used for the pipe roughness calculation:

$$
R_{k}=\frac{8.57 \times 10^{-4}\left(1+2 \gamma / \sqrt{d_{k}}\right)^{2}}{d_{k}^{5}} l_{k} \quad k=1,2, \ldots, n_{p}
$$

where $R_{k}$ is the pipe resistance coefficient, $d_{k}$ is the pipe diameter, $l_{k}$ is the pipe length and $\gamma=0.12$ is the Bazin friction factor. Note that the Bazin formula is commonly used in Italy and the previous study was undertaken using this expression. For the pressure-driven demand model, the value $\mathbf{P}_{\min }=0$ is applied at all nodes while Equation (3) is used for the leakage model.

The larger size network ("Network B", Figure 2) was derived from a real UK system. The network consists of 1991 pipes, 1461 internal nodes and 5 reservoirs (head varying from 40 to 50 m). The diameters used for optimal pipe sizing are those in Table 1 and some statistics of the network are provided in Table 4. The least-cost design was performed by minimising the total design cost subject to a minimum pressure $\mathbf{P}_{\text {ser }}$ (equal to $20 \mathrm{~m}$ for all the nodes) constraint for supplying the design demand $\mathbf{q}_{\text {design }}$ (using $\mathbf{q}_{\text {demand }}=\mathbf{q}_{\text {design }} / 1.25$ ). Finally, the value $\mathbf{P}_{\text {min }}$ was set equal to $10 \mathrm{~m}$ for all the nodes.

\section{NETWORK DESIGN WITH LEAKAGE CONSIDERATIONS}

Network designs obtained from demand-driven analysis assume leakage proportional to nodal demand at each node, but independent of network nodal pressure, leading to unrealistic nodal leakage estimates. For example, two obvious and significant drawbacks of the unrealistic as- 
sumption of a constant leakage percentage normally employed with demand-driven simulation (when combined with optimisation for network design) are:

- the resultant low leakage flows for nodes experiencing low demand, even if they experience high nodal pressure (e.g., are close to a tank).

- the fact that two nodes having equal demand will have equal leakage flow rates associated with them despite their actual hydraulic positions in the network (pressure levels) related to elevations; tank and pump locations, etc..

In order to analyse the effects of the constant-percent leakage assumption, the first step was to calibrate $\beta$ of the leakage model for both networks. To perform this task, the value of $\alpha$ was set to 1.2 and the simulations were performed using $\mathbf{q}_{\text {demand }}$ for both networks, forcing the total leakage flow rate to $25 \%$ of $\mathbf{q}_{\text {demand }}$. The results obtained were $\beta=1.0632 \times 10^{-7}$ and $\beta=2.0748 \times 10^{-8}$ for networks "A" and "B", respectively. The first observation is that, because of the differing total pipe length and average network pressure, $\beta$ differs by one order of magnitude between the networks. This demonstrates that a simulation model taking into account the real $\beta$ is useful even if only used for design purposes because the value for leakage flow rates should be related to the total length, pressure, predicted deterioration, and other factors of the system rather than a constant value (e.g., $25 \%$ used here).

Therefore, the real-losses component of the water balance and/or analysis of night flows based on district metering data (Lambert and Hirner, 2000) can be used for determining comprehensive or zonal values for the leakage flow model parameters in order to plan rehabilitation/expansion of the network. $\beta$ may be seen as a pipe parameter that requires calibration, similar to roughness, 
using as prior information $\beta_{0}$ from water balance or other analyses. For a new network, or a network extension, the concept of unavoidable average real losses (UARL) and infrastructure leakage index (ILI), see Lambert and Hirner (2000), can be used for design purposes to establish a value for $\beta$ instead of using a constant percent coefficient.

Furthermore, Figures 3-6 show the pressure and leakage flow differences for both systems, as computed using the two approaches, demand-driven and pressure-driven simulations. Figures 3 and 5 report the percentage difference in pressure at each node in the network computed with respect to the nodal pressures of the base design case, while Figures 4 and 6 show the nodal leakage flow rates, normalized to the total required network demand, used during the design phase compared to those computed with the pressure-driven simulation. Figures 3 and 5 demonstrate that the majority of the nodes are characterized by a positive pressure difference. This means that the realistic simulation of leakage flow results in a generally higher pressure status than the demand-driven simulation using a constant-percent nodal leakage. The general rise in network pressures is explained by the fact that overall flow throughout pipes has decreased due to greater leakage at nodes with high pressure (e.g., close to tanks).

Figures 4 and 6 present a different outcome of nodal leakage flow computation (referred to total network demand) between the two simulation approaches. Results for "Network A" illustrate how the nodes closest to the tank are characterized by greater leakage flows (pressure-driven simulation), while the most downstream nodes experience lower leakage. More interesting is the situation depicted in Figure 6 related to "Network B". Here, the nodes have been sorted in ascending order according to assigned flow rates (from the design phase) in order to better visual- 
ize the results. Figure 6 gives further evidence of the unrealistic assumption of constant-percent leakage used for design. In fact, there are nodes that have zero, or near zero, demand (and consequently low assumed leakage flow rate), while some (see, for example, the maximum value of $0.5 \%$ of the total network demand) experience a high flow rate as a consequence of high demand. The pressure-driven simulation approach shows more equally distributed nodal leakage flows (around a value close to $0.02 \%$ ), which is a more realistic representation of leakage in this case.

\section{CONVERGENCE AND ROBUSTNESS OF THE SIMULATION MODEL}

Here, the proposed simulation model with pressure-driven demand and leakage is tested for convergence in a steady-state mode. In order to carry out the test, 1,000 simulations were performed for both networks simultaneously varying the following parameters:

- the roughness values for each pipe $\left(R_{k}\right)$.

- the values of each pipe leakage coefficient $\left(\beta_{k}\right)$.

- the value of $\alpha$ applied over the entire network.

The simulations were performed using the values of the above parameters sampled as follows:

- the roughness values for each pipe $\left(R_{k}\right)$ were sampled uniformly from the range of $+/$ $50 \%$ around those related to the optimal values obtained in the network design process (base case).

- the values of each pipe leakage coefficient $\left(\beta_{k}\right)$ were sampled from the range $\left[1 \times 10^{-8}\right.$, $\left.1 \times 10^{-6}\right]$ and $\left[2 \times 10^{-9}, 2 \times 10^{-7}\right]$ for Networks " $A$ " and "B", respectively. The upper and lower boundaries of the sampling range have been selected to be an order of magnitude 
smaller/greater than $\beta$ calibrated in previously described exercise, assuming a water loss of $25 \%$.

- the value of $\alpha$ applied over the entire network was sampled from the range [0.5, 2.5].

Sampling was performed here using Monte-Carlo methodology and, specifically, the Latin Hypercube (LH) technique (McKay et al., 1979) was used as the variance reduction method for limiting the number of samples required for more extensive coverage of the sample space. As indicated, convergence was assumed when $\lambda^{i \text { iter }}$ became less than $10^{-7}$ or the mean of squared errors was less than or equal to $10^{-7}$. A notebook computer with a Pentium Intel M $1.10 \mathrm{GHz}$ processor was used for simulations.

Furthermore, the simulation model was tested without using the over-relaxation parameter $\lambda^{i t e r}$ on Network "B" (the largest one) in order to assess its effectiveness.

Tables 5 and 7 report selected hydraulic parameters for both networks, including flow rates, average network pressure $\left(P_{\text {avg }}\right)$ and number of critical nodes, all revealing the extent of tested conditions.

The first row of Tables 6 and 8 reports the average statistics of the 1,000 simulations considering both the maximum energy/mass balance errors ( $1^{\text {st }}$ and $2^{\text {nd }}$ columns), the mean energy/mass balance errors $\left(3^{\text {rd }}\right.$ and $4^{\text {th }}$ columns $)$, the average number of iterations $\left(5^{\text {th }}\right.$ column $)$ and the average CPU time required for each simulation ( $6^{\text {th }}$ columns). The second row indicates the worst results of the above statistical parameters. Furthermore, Table 8 reports the same statistics $\left(5^{\text {th }}\right.$ and $6^{\text {th }}$ columns) achieved without using the over-relaxation parameter $\lambda^{i t e r}$. They refer to simulations 
performed on "Network B" using the same parameters $\left(R_{k}, \beta_{k}, \alpha\right)$ as in the runs with the overrelaxation parameter $\left(\lambda^{i t e r}\right)$ being used. The statistics were computed on 960 simulations (instead of the 1,000 runs performed) to avoid bias caused by runs experiencing serious convergence problems (10 simulations) or the complete lack of convergence (30 simulations).

The statistics of "Network A" compared with those of the "Network B" (see the maximum errors which are not biased by the network size) prove that by increasing the network size (i.e., number of variables) the algorithm performance does not deteriorate significantly. Furthermore, the statistics in Table 8 for "Network B" show that the achieved maximum errors (about 6 1/s for flow and about $1.8 \mathrm{~cm}$ for pressure) and the average errors (about $0.005 \mathrm{l} / \mathrm{s}$ for flow and about 0.03 $\mathrm{mm}$ for pressure) of the worst simulation (among 1,000) are, for all practical purposes, within the acceptable range. The average number of iterations needed for convergence was about 21 , while the average run time was about 1.8 seconds. The maximum number of iterations was 51 and the maximum run time for a single simulation was about 4.5 seconds.

Furthermore, Figure 7 reinforces the evidence of the algorithm's robustness, showing the number of iterations for each simulation. They are clustered around the values of about 20 , while for only about $3 \%$ of simulations more than 30 iterations were required.

The beneficial influence of the over-relaxation parameter $\lambda^{\text {iter }}$ on convergence of the algorithm was proven experimentally by eliminating problems experienced in $4 \%$ of runs (a very slow convergence for 10 and absence of convergence for 30, out of 1,000 runs). Furthermore, the use of the over-relaxation parameter improves the algorithm run times, but the comparison of the statis- 
tics shows only a slight improvement in the average number of algorithm iterations when using $\lambda^{\text {iter }}$, while the CPU time decreased by approximately $10 \%$. The observation that the single algorithm iteration generally requires less CPU time when using the over-relaxation parameter could be explained by the fact that the solution of the linear system (based on vector $\mathbf{F}$ and matrix $\mathbf{A}$ in the algorithm (7b)) requires less iterations.

In summary, the over-relaxation parameter has proven to be effective for providing robustness to the simulation model. This is explained by the fact that the over-relaxation parameter guides the search for $\mathbf{H}$ and $\mathbf{Q}$ vectors to the most promising regions of the search space. However, the selected initialization (equation 7(a)) influences the algorithm convergence; therefore it is more important for larger networks because of the increase in dimensionality that makes the error surface more complex during the iterative search. Finally, as a secondary effect the over-relaxation parameter seems to generally increase convergence of the linear system solution inside each algorithm iteration (equation (7b)).

\section{CONCLUSIONS}

A new hydraulic simulation model capable of the simultaneous quantification of pressure driven demands and leakage is presented. The steady-state network model was developed by fully integrating system demands (both customer demands and leaks) at the pipe level into the hydraulic model. The importance of an improved leakage model for more realistic network simulation was demonstrated using pipe network design solutions. The comparison of leakage flows for networks designed using demand-driven simulation under the assumption of a constant-percent nodal leakage, shows that the realistic simulation of leakage flow results in generally higher 
pressures across a network than for the demand-driven simulation. The general rise in network pressures for the specific networks (elevations, topology, nodal demands, etc.) is explained by the decrease in overall flow throughout pipes due to greater leakage at nodes with high pressure (e.g., close to tanks). From a numerical standpoint, the algorithm convergence behaviour was tested using one small and one large network derived from two real systems. All the observed convergence/error statistics reinforce the notion that the pressure-driven demand and leakage simulation model developed and presented herein is robust.

\section{Acknowledgment}

Authors wish to thank the Associate Editor and Reviewers for their thorough and insightful review of our manuscript. The reviews have proven to be particularly important for improving the quality of our article.

\section{APPENDIX I. REFERENCES}

Ackley, J.R.L., Tanyimboh, T.T., Tahar, B., and Templeman, A.B. (2001). "Head-driven analysis of water distribution systems." Ulanicki, B.(ed.) Proceedings of Computer and Control in Water Industry (CCWI), Water software systems: theory and applications, Research Studies Press, England, Vo.1, 183-192.

Ainola, L., Koppel, T., Tiiter. T., and Vassiljev, A. (2000). "Water Network Model Calibration Based on Grouping Pipes with Similar Leakage and Roughness Estimates.” Proceedings of the Joint Conference on Water Resource Engineering and Water Resource Planning and Management, (EWRI), Minneapolis, MN, 104, 197. 
Almandoz, J., Cabrera, E.M., Arregui, F., Cabrera, E.Jr., and Cobacho, R. (2005). "Leakage Assessment through Water Distribution Network Simulation." J. Water Resour. Plan. and Manage., 131(6), 458-466.

Berardi, L., Savic, D.A., and Giustolisi, O. (2005). "Investigation of burst-prediction formulas for water distribution systems by evolutionary computing." Proceedings of Computer and Control in Water Industry (CCWI), Vo.2, 275-280.

Chandapillai, J. (1991). "Realistic simulation of water distribution system." J. of Transportation Eng., 117(2), 258-263.

Colombo, A.F., and Karney, B.W. (2002). "Energy and costs of leaky pipes: Toward a comprehensive Picture.” J. Water Resour. Plan. Manage., 128(6), 441-450.

Germanopoulos, G. (1985). "A technical note on the inclusion of pressure dependent demand and leakage terms in water supply network models." Civil Engineering Systems, 2(September), 171-179.

Germanopoulos, G., and Jowitt, P.W. (1989). "Leakage reduction by excessive pressure minimization in a water supply network." Proc., Instn. Civ. Engrs., Part 2, 87(June), 195-214.

Gupta, R., and Bhave, P.R. (1996). “Comparison of methods for predicting deficient-network performance.” J. Water Resour. Plan. and Manage., 122(3), 214-217.

Kalungi, P., and Tanyimboh, T. (2003). "Redundancy model for water distribution systems." Reliability Engineering \& System Safety, 82(3), 275-286.

Kapelan, Z., Savic, D.A., and Walters, G.A. (2005). "Multiobjective Design of Water Distribution Systems under Uncertainty.” Water Resources Research, 41(11), W11407.

Kettler, A.J., and Goulter, I.C. (1985). “An analysis of pipe breakage in urban water distribution networks." Can. J. Civ. Eng., 12, 286-293. 
Kleiner, Y., and Rajani, B.B. (1999). "Using limited data to assess future needs." J. Am. Water Works Assn., 91(7), 47-62.

Kleiner, Y., and Rajani, B.B. (2001). "Comprehensive review of structural deterioration of water mains: Statistical models." Urban Water, 3(3), 121-150.

Kleiner, Y., and Rajani, B.B. (2002). "Forecasting Variations and Trends in Water-Main Breaks.” J. Infrastr. Sys., 8(4), 122-131.

Jowitt, P.W., and Xu, C. (1990). “Optimal Valve Control in Water Distribution Networks.” J. Water Resour. Plan. and Manage., 126 July/August, 455-472.

Lambert, A.O. (1994). “Accounting for losses: the bursts and background concept (BABE)." Institution of Water and Environment Management Journal, 8(2), 205-214

Lambert, A.O. (2001). "What do we know about Pressure: Leakage Relationships in Distribution Systems ?"IWA Conference on System Approach to Leakage Control and Water Distribution Systems Management, Brno, ISBN 80-7204-197-5.

Lambert, A.O., and Hirner, W. (2000). “The Blue Pages.” IWA Publishing, London, UK.

May, J., (1994). “Pressure dependent leakage.” World Water \& Environmental Engineering Management, October.

Male, W., Walski, T.M. and Slutsky, A.H. (1990). "Analysing water main replacement policies.” J. Water Resour. Plan. and Manage., 116(3), 362-375.

McKay, M.D., Conover, W.J., and Beckman, R.J. (1979). “A Comparison of Three Methods for Selecting Values of Input Variables in the Analysis of Output from a Computer Code." Technometrics, 211, 239-245.

Rossman, L.A. (2000). “EPANET 2 users manual.” U.S. Environmental Protection Agency, Cincinnati, Ohio. 
Savic, D.A., and Walters, G.A. (1997). "Genetic Algorithms for the Least-cost Design of Water Distribution Networks.” J. Water Resour. Plan. and Manage., 123(2), 67-77.

Shamir, U., and Howard, C.D.D. (1979). "An analytic approach to scheduling pipe replacement.”, J. Am. Water Works Assn., 117(5), 248-258.

Todini, E. (2003). "A more realistic approach to the "extended period simulation" of water distribution networks." Advances in Water Supply Management, Maksimovic C., Butler D., and Memon F.A. (eds), A.A. Balkema Publishers, Lisse, The Netherlands, 173-184.

Todini, E., and Pilati, S. (1988). "A gradient algorithm for the analysis of pipe networks.” Computer Applications in Water Supply, Coulbeck B., and Orr C.H. (eds), Vo.1 (System analysis and simulation), John Wiley \& Sons, London, UK, 1-20.

Vairavamoorthy, K., and Lumbers, J. (1998). "Leakage Reduction in Water Distribution Systems: Optimal Valve Control.” J. Hydr. Eng., 124(9), 1146-1154.

Wagner, J.M., Shamir, U., and Marks, D.H. (1988). "Water distribution reliability: simulation methods.” J. Water Resour. Plan. and Manage., 114(3), 276-294.

Walski, T.M. (1987). “Replacement rules for water mains.” J. Am. Water Works Assn., 79(9), 3338.

Walski, T.M., and Pelliccia, A. (1982). "Economics analysis of water main breaks." J. Am. Water Works Assn., 74(3), 140-147.

Wu, Z.Y., Wang, R.H., Walski, T.M., Yang, S.Y., and Boudler, D. (2006). "Efficient pressure dependent demand model for large water distribution system analysis." $8^{\text {th }}$ Water Distribution System Analysis Symposium, Cincinnati, Ohio, USA, August 27-30, (CD-ROM). 


\section{APPENDIX II. NOTATION}

The following symbols are used in the paper:

$\mathbf{A}=$ temporary matrix used in simulation model algorithm;

$\mathbf{A}_{n n}=$ diagonal matrix whose elements are from the scalar product $-\left(\mathbf{q}_{a c t}+\mathbf{q}_{l}\right) \mathbf{H}^{-1}$;

$\overline{\mathbf{A}}_{p n} \quad=\quad$ general topological matrix;

$\mathbf{A}_{p n}, \mathbf{A}_{n p}=\quad$ topological incidence sub-matrices;

$\mathbf{A}_{p p}=$ diagonal matrix whose elements are $R_{k}\left|Q_{k}\right|^{n-1}$;

age $=$ age of the pipe in the statistical model of pipe failure;

$C_{k}=\quad$ coefficient for burst leakage model for $k$-th pipe;

$d / d_{k}=\quad$ diameter of the $k$-th pipe;

$\mathbf{D}_{n n}, \quad=\quad$ diagonal matrices whose elements are derivatives of $\mathbf{q}_{a c t}$ elements;

$\mathbf{D}_{p p}, \quad=\quad$ diagonal matrices whose elements are derivatives of $R_{k}\left|Q_{k}\right|^{n-1} Q_{k}$;

$\mathbf{D L}_{n n}=$ diagonal matrices whose elements are derivatives of $\mathbf{q}_{l}$ elements.

$\mathbf{F} \quad=\quad$ temporary matrix used in Todini's algorithm;

$\mathbf{H}=$ vector of total network heads;

$\mathbf{H}_{0}=\quad$ vector of total fixed (i.e. known) network heads;

$\mathbf{I}_{n n}, \mathbf{I}_{p p}=\quad$ identity matrices;

$i=$ matrix index for nodes;

$k=$ matrix index for pipes;

$l / l_{k}=\quad$ length of the $k$-th network pipe;

$n=$ head loss equation exponent and sub-matrix index;

$n_{0} \quad=\quad$ total number of known heads;

$n_{p} \quad=\quad$ total number of network pipes; 


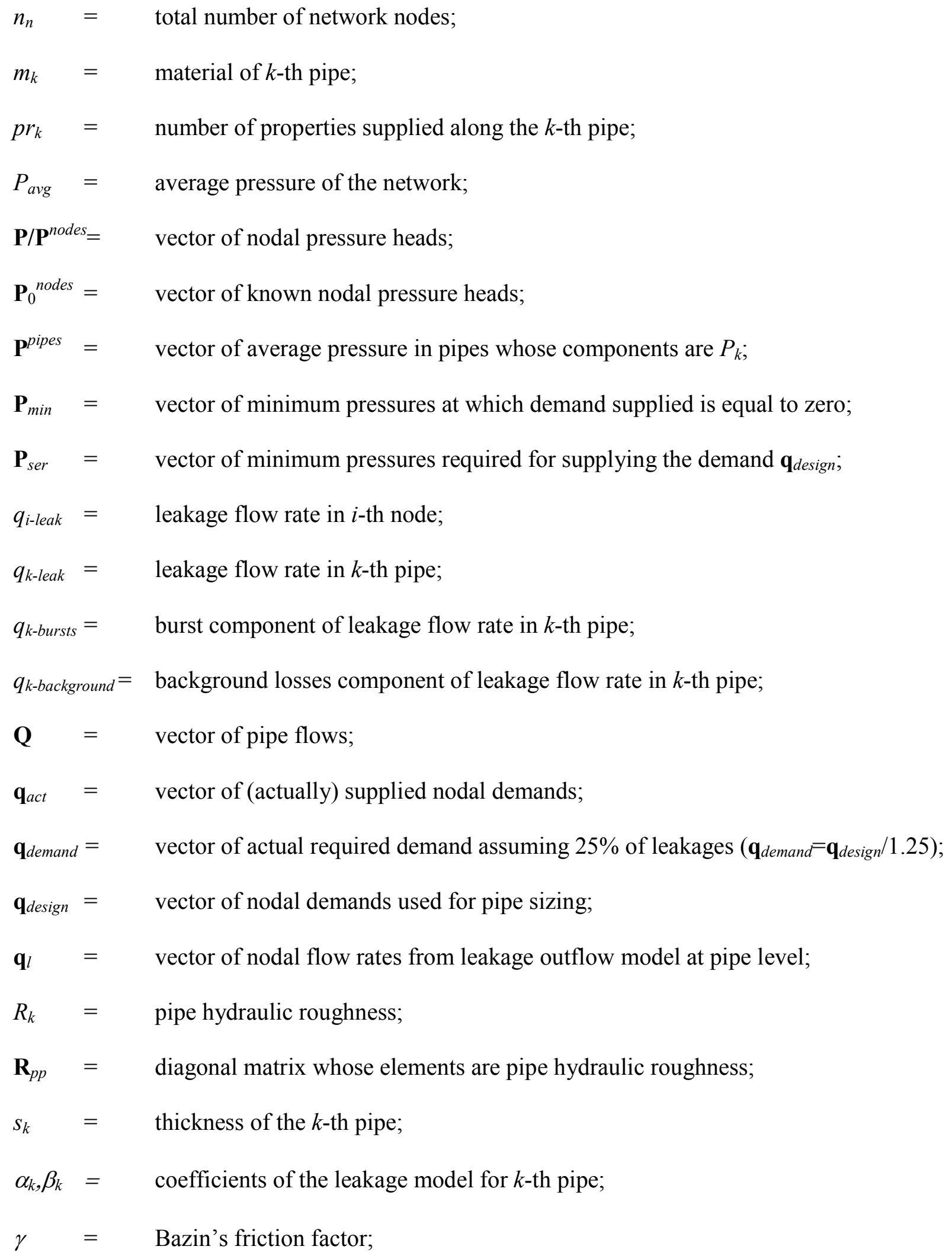


$\Lambda_{n n}, \Lambda_{p p}=\quad$ diagonal matrices of over-relaxation coefficients;

$\lambda^{\text {iter }}=$ step size or over-relaxation coefficient at iter-th iteration;

Operators and Acronyms:

()$^{\mathrm{T}}=$ vector/matrix transpose operator;

diag $=$ diagonal elements of a square matrix: 
List of Tables

Table 1. Diameters used for pipe sizing of network "A" and "B".

Table 1. "Network A" pipe data.

Table 3. "Network A" node data.

Table 4. The range of some parameters of the "Network B".

Table 5. Minimum/maximum value of some useful parameters during leakage-driven simulations. $N_{C R}$ is the number of critical nodes $\left(P_{i}<P_{i \text {-ser }}\right)$.

Table 6. Statistics of leakage-driven simulations.

Table 7. Minimum/maximum value of some useful parameters during leakage-driven simulations. $N_{C R}$ is the number of critical nodes $\left(P_{i}<P_{i \text {-ser }}\right)$.

Table 8. Statistics of leakage-driven simulations. 
Table 1. Diameters used for pipe sizing of networks "A" and "B".

\begin{tabular}{|c|c|}
\hline $\begin{array}{c}d \\
(\mathrm{~m})\end{array}$ & $\begin{array}{c}R / l \\
\left(\mathrm{~s}^{2} / \mathrm{m}^{6}\right)\end{array}$ \\
\hline 0.100 & 265.147 \\
\hline 0.164 & 18.565 \\
\hline 0.184 & 9.882 \\
\hline 0.204 & 5.629 \\
\hline 0.229 & 3.068 \\
\hline 0.258 & 1.639 \\
\hline 0.290 & 0.867 \\
\hline 0.327 & 0.460 \\
\hline 0.368 & 0.247 \\
\hline 0.500 & 0.049 \\
\hline 0.750 & 0.006 \\
\hline 1.000 & 0.001 \\
\hline
\end{tabular}


Table 2. "Network A" pipe data.

\begin{tabular}{|c|c|c|c|c|}
\hline $\begin{array}{c}\text { pipe } \\
\text { No }\end{array}$ & $\begin{array}{c}\text { start } \\
\text { node }\end{array}$ & $\begin{array}{c}\text { end } \\
\text { node }\end{array}$ & $\begin{array}{c}l_{k} \\
(\mathrm{~m})\end{array}$ & $\begin{array}{c}d_{k} \\
(\mathrm{~m})\end{array}$ \\
\hline 1 & 1 & 2 & 348.5 & 0.327 \\
\hline 2 & 2 & 3 & 955.7 & 0.290 \\
\hline 3 & 3 & 4 & 483 & 0.100 \\
\hline 4 & 3 & 9 & 400.7 & 0.290 \\
\hline 5 & 2 & 4 & 791.9 & 0.100 \\
\hline 6 & 1 & 5 & 404.4 & 0.368 \\
\hline 7 & 5 & 6 & 390.6 & 0.327 \\
\hline 8 & 6 & 4 & 482.3 & 0.100 \\
\hline 9 & 9 & 10 & 934.4 & 0.100 \\
\hline 10 & 11 & 10 & 431.3 & 0.184 \\
\hline 11 & 11 & 12 & 513.1 & 0.100 \\
\hline 12 & 10 & 13 & 428.4 & 0.184 \\
\hline 13 & 12 & 13 & 419 & 0.100 \\
\hline 14 & 22 & 13 & 1023.1 & 0.100 \\
\hline 15 & 8 & 22 & 455.1 & 0.164 \\
\hline 16 & 7 & 8 & 182.6 & 0.290 \\
\hline 17 & 6 & 7 & 221.3 & 0.290 \\
\hline 18 & 1 & 19 & 583.9 & 0.164 \\
\hline 19 & 5 & 18 & 452 & 0.229 \\
\hline 20 & 6 & 16 & 794.7 & 0.100 \\
\hline 21 & 7 & 15 & 717.7 & 0.100 \\
\hline 22 & 8 & 14 & 655.6 & 0.258 \\
\hline 23 & 15 & 14 & 165.5 & 0.100 \\
\hline 24 & 16 & 15 & 252.1 & 0.100 \\
\hline 25 & 17 & 16 & 331.5 & 0.100 \\
\hline 26 & 18 & 17 & 500 & 0.204 \\
\hline 27 & 17 & 21 & 579.9 & 0.164 \\
\hline 28 & 19 & 23 & 842.8 & 0.100 \\
\hline 29 & 21 & 20 & 792.6 & 0.100 \\
\hline 30 & 20 & 14 & 846.3 & 0.184 \\
\hline 31 & 9 & 11 & 164 & 0.258 \\
\hline 32 & 23 & 21 & 427.9 & 0.100 \\
\hline 33 & 19 & 18 & 379.2 & 0.100 \\
\hline 34 & 24 & 1 & 158.2 & 0.368 \\
\hline & & & & \\
\hline
\end{tabular}


Table 3. "Network A" node data.

\begin{tabular}{|c|c|c|c|}
\hline $\begin{array}{c}\text { node } \\
\mathrm{ID}\end{array}$ & \begin{tabular}{c}
$q_{\text {i-design }}(\mathrm{l} / \mathrm{s})$ \\
\hline 1
\end{tabular} & $\begin{array}{c}H L_{i} \\
(\mathrm{~m})\end{array}$ & $\begin{array}{c}P_{i} \\
(\mathrm{~m})\end{array}$ \\
\hline 2 & 17.034 & 6.4 & 26.90 \\
\hline 3 & 14.947 & 6 & 24.81 \\
\hline 4 & 14.280 & 8.4 & 21.30 \\
\hline 5 & 10.133 & 7.4 & 23.22 \\
\hline 6 & 15.35 & 9 & 20.10 \\
\hline 7 & 9.114 & 9.1 & 18.91 \\
\hline 8 & 10.510 & 9.5 & 17.90 \\
\hline 9 & 12.182 & 8.4 & 17.85 \\
\hline 10 & 14.579 & 10.5 & 12.66 \\
\hline 11 & 9.0072 & 9.6 & 16.23 \\
\hline 12 & 7.5745 & 11.7 & 10.12 \\
\hline 13 & 15.200 & 12.3 & 10.03 \\
\hline 14 & 13.550 & 10.6 & 15.41 \\
\hline 15 & 9.226 & 10.1 & 14.00 \\
\hline 16 & 11.200 & 9.5 & 14.36 \\
\hline 17 & 11.469 & 10.2 & 15.30 \\
\hline 18 & 10.818 & 9.6 & 18.83 \\
\hline 19 & 14.675 & 9.1 & 19.35 \\
\hline 20 & 13.318 & 13.9 & 10.01 \\
\hline 21 & 14.631 & 11.1 & 11.48 \\
\hline 22 & 12.012 & 11.4 & 14.00 \\
\hline 23 & 10.326 & 10 & 10.45 \\
\hline 24 & & 15 & $\mathrm{H}_{01}=21.4+15$ \\
\hline
\end{tabular}


Table 4. The range of some parameters of the "Network B".

\begin{tabular}{|c|c|c|c|}
\hline & $\begin{array}{c}l_{k} \\
(\mathrm{~m})\end{array}$ & $\begin{array}{c}q_{i-\text {-design }} \\
(1 / \mathrm{s})\end{array}$ & $\begin{array}{c}R_{k} \\
\left(\mathrm{~s}^{2} / \mathrm{m}^{5}\right)\end{array}$ \\
\hline Total/Mean & 486927 & 3183.4 & 6155.6 \\
\hline Minimum & 1 & 0 & 0.0013 \\
\hline Maximum & 2530 & 63 & 530293.3 \\
\hline
\end{tabular}


Table 5. Minimum/maximum value of some useful parameters during leakage-driven simulations. $N_{C R}$ is the number of critical nodes $\left(P_{i}<P_{i-s e r}\right)$.

\begin{tabular}{|c|c|c|c|c|c|}
\hline "Network A" & $\mathbf{q} / \mathbf{q}_{\text {demand }}$ & $N_{C R}$ & $\begin{array}{c}\mathbf{q}_{\text {act }} \\
\left(\mathrm{m}^{3} / \mathrm{s}\right)\end{array}$ & $\begin{array}{c}\mathbf{q}_{/} \\
\left(\mathrm{m}^{3} / \mathrm{s}\right)\end{array}$ & $\begin{array}{c}P_{\text {avg }} \\
(\mathrm{m})\end{array}$ \\
\hline $\begin{array}{c}\text { Minimum among the } \\
\text { 1,000 simulations }\end{array}$ & 0.1501 & 0 & 0.1005 & 0.0339 & 2.78 \\
\hline $\begin{array}{c}\text { Average among the } \\
1,000 \text { simulations }\end{array}$ & 1.1833 & 13 & 0.1803 & 0.2669 & 9.61 \\
\hline $\begin{array}{c}\text { Maximum among the } \\
1,000 \text { simulations }\end{array}$ & 3.4701 & 22 & 0.2256 & 0.7829 & 19.81 \\
\hline
\end{tabular}


Table 6. Statistics of leakage-driven simulations.

\begin{tabular}{|c|c|c|c|c|c|c|}
\hline \multirow{2}{*}{ "Network A" } & \multicolumn{2}{|c|}{ Maximum error } & \multicolumn{2}{c|}{ Mean error } & \multicolumn{2}{c|}{} \\
\cline { 2 - 6 } & $\begin{array}{c}\text { Energy balance } \\
(\mathrm{m})\end{array}$ & $\begin{array}{c}\text { Mass balance } \\
\left(\mathrm{m}^{3} / \mathrm{s}\right)\end{array}$ & $\begin{array}{c}\text { Energy balance } \\
(\mathrm{m})\end{array}$ & $\begin{array}{c}\text { Mass balance } \\
\left(\mathrm{m}^{3} / \mathrm{s}\right)\end{array}$ & $\begin{array}{c}\text { Iteration } \\
\text { number }\end{array}$ & $\begin{array}{c}\text { CPU time } \\
(\mathrm{s})\end{array}$ \\
\hline $\begin{array}{c}\text { Average among the } \\
1,000 \text { simulations }\end{array}$ & $4.50 \times 10^{-4}$ & $1.63 \times 10^{-4}$ & $3.82 \times 10^{-5}$ & $2.49 \times 10^{-5}$ & 9.84 & 0.012 \\
\hline $\begin{array}{c}\text { Worst among the } \\
1,000 \text { simulations }\end{array}$ & $2.24 \times 10^{-3}$ & $1.44 \times 10^{-3}$ & $2.11 \times 10^{-4}$ & $1.64 \times 10^{-4}$ & 25 & 0.047 \\
\hline
\end{tabular}


Table 7. Minimum/maximum value of some useful parameters during leakage-driven simulations. $N_{C R}$ is the number of critical nodes $\left(P_{i}<P_{i-s e r}\right)$.

\begin{tabular}{|c|c|c|c|c|c|}
\hline "Network B" & $\mathbf{q} / \mathbf{q}_{\text {demand }}$ & $N_{C R}$ & $\begin{array}{c}\mathbf{q}_{a c t} \\
\left(\mathrm{~m}^{3} / \mathrm{s}\right)\end{array}$ & $\begin{array}{c}\mathbf{q}_{/} \\
\left(\mathrm{m}^{3} / \mathrm{s}\right)\end{array}$ & $\begin{array}{c}P_{\text {avg }} \\
(\mathrm{m})\end{array}$ \\
\hline $\begin{array}{c}\text { Minimum among the } \\
\text { 1,000 simulations }\end{array}$ & 0.1110 & 0 & 0.1381 & 0.2826 & 6.70 \\
\hline $\begin{array}{c}\text { Average among the } \\
1,000 \text { simulations }\end{array}$ & 2.0197 & 708 & 1.5709 & 5.1436 & 19.18 \\
\hline $\begin{array}{c}\text { Maximum among the } \\
\text { 1,000 simulations }\end{array}$ & 5.5924 & 1225 & 2.5467 & 14.2421 & 33.62 \\
\hline
\end{tabular}


Table 8. Statistics of leakage-driven simulations.

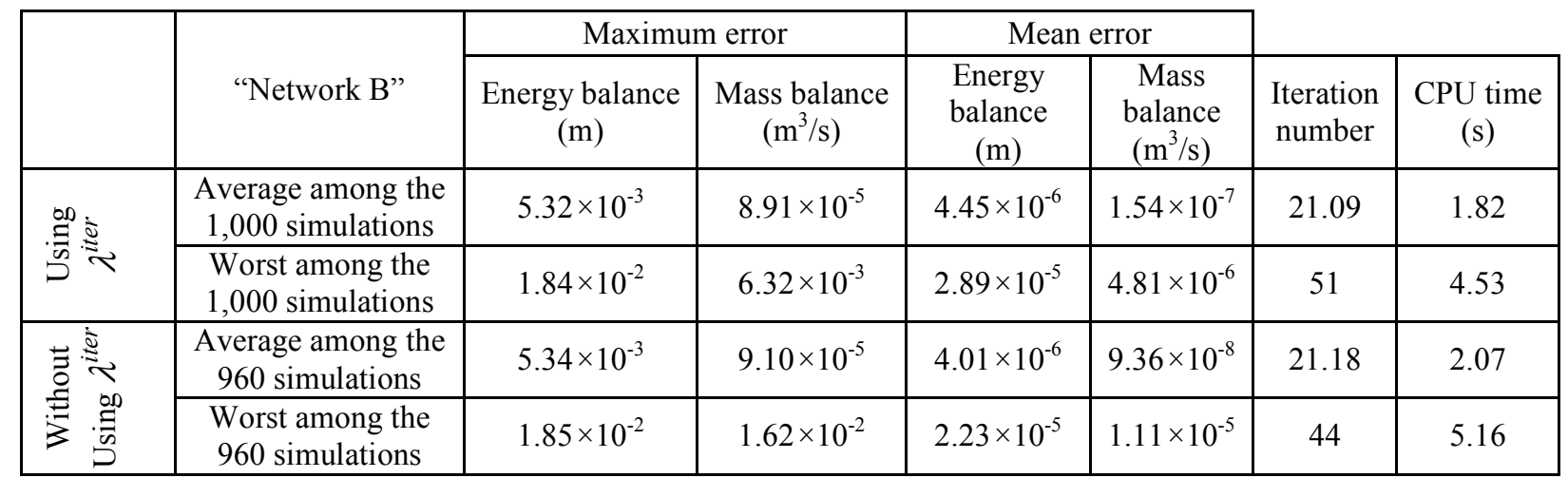




\section{LIST OF FIGURES}

Figure 1. Layout of "Network A".

Figure 2. Layout of "Network B".

Figure 3. Nodal pressure difference (in percentage of the nodal design pressure) between the two simulation approaches ("Network A").

Figure 4. Percentage (referring to total network demand) of nodal leakage flow of the two simulation approaches ("Network A").

Figure 5. Nodal pressure difference (in percentage of the nodal pressure of design) between the two simulation approaches ("Network B").

Figure 6. Percentage (referring to total network demand) of nodal leakage flow of the two simulation approaches ("Network B").

Figure 7. Number of iterations for convergence for each simulation ("Network B"). 